\title{
Plasma synthesis of nanostructures for improved thermoelectric properties
}

Nils Petermann ${ }^{1}$, Niklas Stein ${ }^{2}$, Gabi Schierning ${ }^{2,4}$, Ralf Theissmann ${ }^{2,4}$, Benedikt Stoib ${ }^{3}$, Christian Hecht $^{1}$, Christof Schulz ${ }^{1,4}$, Hartmut Wiggers ${ }^{1,4}$

${ }^{1}$ Institut für Verbrennung und Gasdynamik, Universität Duisburg-Essen, 47057 Duisburg, Germany

${ }^{2}$ Nanostrukturtechnik, Universität Duisburg-Essen, Bismarckstr. 81, 47057 Duisburg, Germany

${ }^{3}$ Walter Schottky Institut, Technische Universität München, Am Coulombwall 3, 85748 Garching

${ }^{4}$ CeNIDE, Center for NanoIntegration Duisburg-Essen, 47057 Duisburg

e-mail: hartmut.wiggers@uni-due.de

\section{Abstract}

The utilization of silicon-based materials for thermoelectrics is studied with respect to synthesis and processing of doped silicon nanoparticles from gas phase plasma synthesis. It is found that plasma synthesis enables for the formation of spherical, highly crystalline and soft-agglomerated materials. We discuss the requirements for the formation of dense sintered bodies while keeping the crystallite size small. Both, small particles sizing a few ten nanometer and below that are easily achievable from plasma synthesis, and a weak surface oxidation lead to a pronounced sinter activity about $350 \mathrm{~K}$ below the temperature usually needed for successful densification of silicon. The thermoelectric properties of our sintered materials are comparable with the best results found for nanocrystalline silicon prepared by other methods than plasma synthesis. 


\section{Introduction}

The continuous and increasing discussion regarding possibilities for energy harvesting, power efficiency and sustainability has led to an intensive (re)search for further development and optimization of energy conversion processes. This covers all areas from improvement in efficiency over improved insulation techniques to waste heat recovery. The latter one becomes more and more interesting as new approaches in thermoelectrics pave the way for its commercialization. Thermoelectric materials are solids which allow for a direct conversion of temperature differences into electrical energy. Thermoelectric generators have proven to show long-term stability and reliability even under extreme conditions but up to now they are mainly used in niche applications like space probes ${ }^{1}$ or at remote locations ${ }^{2}$. Despite of still relatively low over-all performance with respect to costs and efficiency, thermoelectrics are highly interesting for heat recovery, especially at hightemperatures such as waste heat from processing plants or from automobile exhaust, containing about $30 \%$ of the overall energy delivered from the fuel ${ }^{3}$. Therefore, thermoelectric generators mounted at the exhaust pipe of combustion engines have the potential to significantly improve the fuel economy through conversion of waste heat to electric energy which is increasingly required to drive the electrical components of modern cars and will be even more important as an energy source in hybrid cars.

\section{Thermoelectrics}

Thermoelectric generators are operated based on the Seebeck effect. It describes the emergence of a thermo voltage, when the ends of an electrically conductive material are exposed to different temperatures.

$$
U=S \Delta T
$$

Equation 1

$S$ is the Seebeck-coefficient or thermo power, $U$ the thermo voltage, and $\Delta T$ the temperature difference. The origin of the thermo voltage is a thermally driven diffusion of charge carriers from the hot to the cold end of the chunk. Thus, an electrical field is generated with the polarity of the majority charge carriers at the cold side. Hence, semiconductors can be used to transform temperature gradients into usable electrical energy. The efficiency of a thermoelectric generator is limited to a fraction of the 
Carnot efficiency, determined by the dimensionless figure of merit $Z T$ defined as

$$
Z T=\frac{S^{2} \sigma}{\kappa_{e l}+\kappa_{\text {lattice }}} \cdot T
$$

Equation 2

where $\sigma$ is the electrical conductivity, while $\kappa_{e l}$ and $\kappa_{\text {lattice }}$ are the electronic and the lattice part of the thermal conductivity, respectively. The numerator $S^{2} \sigma$ is called the power factor.

For decades, the main approach towards advanced thermoelectric materials was focused on the development of degenerate semiconductor materials like $\mathrm{Bi}_{2} \mathrm{Te}_{3}, \mathrm{Sb}_{2} \mathrm{Te} 3, \mathrm{PbTe}$, and alloys such as . $\mathrm{Si}_{\mathrm{x}} \mathrm{Ge}_{1-\mathrm{x}}$. This led to reasonable values for $Z T$ with peak values typically in the range of 0.8 to 1.1. From the mid 1990s, efforts towards an improved performance of thermoelectric materials have concentrated to reduce thermal conductivity. Note that the electron thermal conductivity is proportional to the electrical conductivity, so ZT can not be increased by decreasing the electron thermal conductivity

The lattice thermal conductivity - responsible for heat transport by phonons/lattice vibrations - is directly related to the mean free path of phonons. From detailed microscopic investigations it was found that high efficiency thermoelectrics feature structures in the nanometer range. As a result, scientists started to further enhance the efficiency of thermoelectric materials by introduction of artificial nanostructures to synthesize materials with a significantly lowered thermal conductivity. According to ISI Web of Science, the number of both, publications and citations with keywords nano* and thermoelectric* have experienced an impressive boom. The number of publications rose from 8 in 1996 to 290 in 2009 and the number of citations from 22 in 1996 to 4093 in 2009.

In this contribution, we focus on the formation of artificial nanostructures and grain boundaries as a highly promising and powerful tool to introduce enhanced phonon scattering mechanisms into the material. The goal is to lower its thermal conductivity while simultaneously preventing a decrease in electronic conductivity. Hence, the structure size has to be adapted between the electron wavelength and the phonon mean free path. While the electron mean free path is in the order of some nanometers, typical phonon mean free paths are in the range of up to several $100 \mathrm{~nm}$. Especially low-frequency acoustic phonons, which carry most heat due to their high group velocity, show mean free paths up to 
some microns ${ }^{4}$. Thus, tailoring nanostructures in an appropriate size can effectively scatter phonons while having less impact on the charge carrier transport properties.

Nanometer-scaled materials may be formed by compacting nanoparticles under preservation of the nanostructure which is the approach of this work. Other possibilities include intrinsic structural modulations at the nanometer scale ${ }^{5,6}$, the incorporation of precipitates and inclusions ${ }^{5,7}$, or the use of other obstacles for phonons like structural domains ${ }^{8}$. Further strategies to tune the thermal conductivity of thermoelectric materials are the incorporation of rattling atoms with large vibrational amplitudes at partially filled structure sites 9,10 and the transition to complex crystal structures where the number of optical phonon modes is increased and - as a consequence - the heat transport by the acoustic phonon branches reduced ${ }^{11}$. Alloying produces disorder at the atomic scale so that the thermal conductivity is at the 'alloy limit' ${ }^{12}$ and may be combined with nano structuring in order to affect the complete phonon spectrum ${ }^{13-15}$. Remarkably high values of the figure of merit can be achieved in isolated nanostructures ${ }^{16,17}$ and multilayer structures ${ }^{18}$ which combine low values of the thermal conductivity and optimized electronic structure. For those multilayer structures, expensive fabrication techniques are employed like lithography or molecular beam epitaxy, and possible routes towards a large scale device fabrication are a long way off. Nevertheless, plasma assisted processes like plasma-enhanced chemical vapor deposition (PECVD), atomic layer deposition (PEALD), and sputtering are highly promising techniques that might also be suitable for commercial multilayer synthesis.

With respect to waste heat harvesting from combustion engines, the materials of interest have to fulfill several requirements: i) the thermal stability of possible materials has to cover a temperature range from 300 to $900{ }^{\circ} \mathrm{C}$; ii) the materials should consist of abundant and - preferably - non-toxic materials; iii) the devices should consist of $\mathrm{p}$ - and n-type doped materials with similar thermal expansion coefficient for high thermal stability. According to ${ }^{19}$ the most promising materials in the required temperature range are $\mathrm{CoSb}_{3},(\mathrm{GeTe})_{0.85}(\mathrm{AgSbTe} 2)_{0.15}(\mathrm{TAGS}), \mathrm{CeFe}_{4} \mathrm{Sb}_{12}, \mathrm{Yb}_{14} \mathrm{MnSb}_{11}$, and $\mathrm{Si}_{\mathrm{x}} \mathrm{Ge}_{1-\mathrm{x}}$. To our opinion, the most promising high temperature material with respect to the requirements mentioned above is $\mathrm{Si}_{\mathrm{x}} \mathrm{Ge}_{1-\mathrm{x}}$ and it has been shown, that nanostructuring of $\mathrm{Si}$ and $\mathrm{Si}_{\mathrm{x}} \mathrm{Ge}_{1-\mathrm{x}}$ is a successful way towards enhanced thermolectric properties ${ }^{20,21}$. 
The thermoelectric performance of bulk silicon is poor, as it exhibits diamond structure and therefore, its bulk thermal conductivity is quite high (about $140 \mathrm{~W} \mathrm{~m}^{-1} \mathrm{~K}^{-1}$ at ambient temperature ${ }^{22}$ ). The high value of $\kappa$ limits the efficiency of bulk silicon as a thermoelectric material, but this material will become an interesting candidate as a basis for widely-spread thermoelectric applications if its thermal conductivity could be significantly reduced as described above.

Decades ago, Dismukes et al. reported a thermal conductivity of about $5 \mathrm{~W} \mathrm{~m}^{-1} \mathrm{~K}^{-1}$ for $\mathrm{n}$-type $\mathrm{Si}_{0.8} \mathrm{Ge}_{0.2}$ alloys at room temperature with a peak $Z T=1$ for the n-type alloy at $1200^{\circ} \mathrm{C}^{23}$. Alloys of silicon-germanium have proven to show a much lower thermal conductivity than pure silicon, which is related to alloy scattering of phonons, resulting in a much better thermoelectric performance ${ }^{24}$. Microstructured SiGe alloys are in use in radioisotope thermoelectric generators (RTG) to power several space probes ${ }^{1}$. Alloying silicon with germanium is thus one way to lower thermal conductivity and to achieve good $Z T$ values, but germanium is a rare and expensive material. Nevertheless, using germanium in low concentration could be an optimum with respect to costs and efficiency. Zhu et al. ${ }^{14}$ reported about nanostructured $\mathrm{Si}_{0.95} \mathrm{Ge}_{0.05}$ alloys with a significantly enhanced power factor, especially at room temperature, and a low thermal conductivity resulting in a similar figure of merit as found for the aforementioned $\mathrm{Si}_{0.8} \mathrm{Ge}_{0.2}$ bulk alloys. Additionally, many other materials with promising thermoelectric properties consisting of highly available and sustainable compounds also contain silicon as a main component such as iron-, manganese- and magnesium silicides. Composite materials consisting of $\mathrm{SiGe}$ with embedded nanocrystalline silicides are discussed as high performance materials. In summary, nanostructuring of silicon-based materials has the potential to be used for high temperature thermoelectrics enabling for a reasonable and cost-efficient utilization.

\section{Synthesis of nanosized materials}

There are two main strategies to synthesize the required nanostructured materials, the top-down and the bottom-up approach. The top-down method starts from bulk materials by grinding ${ }^{20,21}$ or highly specified methods like spark erosion or laser ablation ${ }^{15,25}$. Generally, these methods end up in nanostructured powder with a very broad particle size distribution and in most cases, these methods exhibit a specific lower limit concerning the particle size, which is in the range of a few ten nanometers. While the scattering rate of phonons is directly related to the number of grain boundaries, 
it is necessary to achieve a very small particle size to maximize the reduction of phonon thermal conductivity and methods other than top-down ones are required. As the formation of high performance thermoelectrics requires degenerate semiconductors with a very high content of dopants, these dopants must also be added as bulk material during formation of the nanosized powder, which might be difficult or even impossible for top-down methods.

The bottom-up approaches start from liquid or gaseous precursor materials ${ }^{26,27}$. These precursors react or decompose resulting in nucleation, coagulation and growth of nanoparticles. Depending on the reactor concept, thermodynamic as well as kinetic control of particle formation can be realized. As the time constants in liquid phase synthesis are usually much higher than in gas phase synthesis, liquid phase usually leads to thermodynamically controlled products, while gas phase synthesis enables for kinetic control. Moreover, liquid phase synthesis requires the addition of stabilizing, surface-active compounds while gas phase synthesis usually leads to high-purity products. Additionally, it is favored for the formation of non-oxidic nano materials because of its flexibility and scalability ${ }^{28}$. It is an established method for the formation of silicon nanoparticles ${ }^{29,30}$ and has also been used recently for the formation of germanium nanoparticles ${ }^{31}$. High purity can be obtained as gases are available with a very low degree of impurities and particle synthesis can be carried out under high purity conditions. Control over stoichiometry, doping and residence time is attained as long as vaporizable precursor materials are available while mass flow controllers allow for a precise metering of the process gases. 

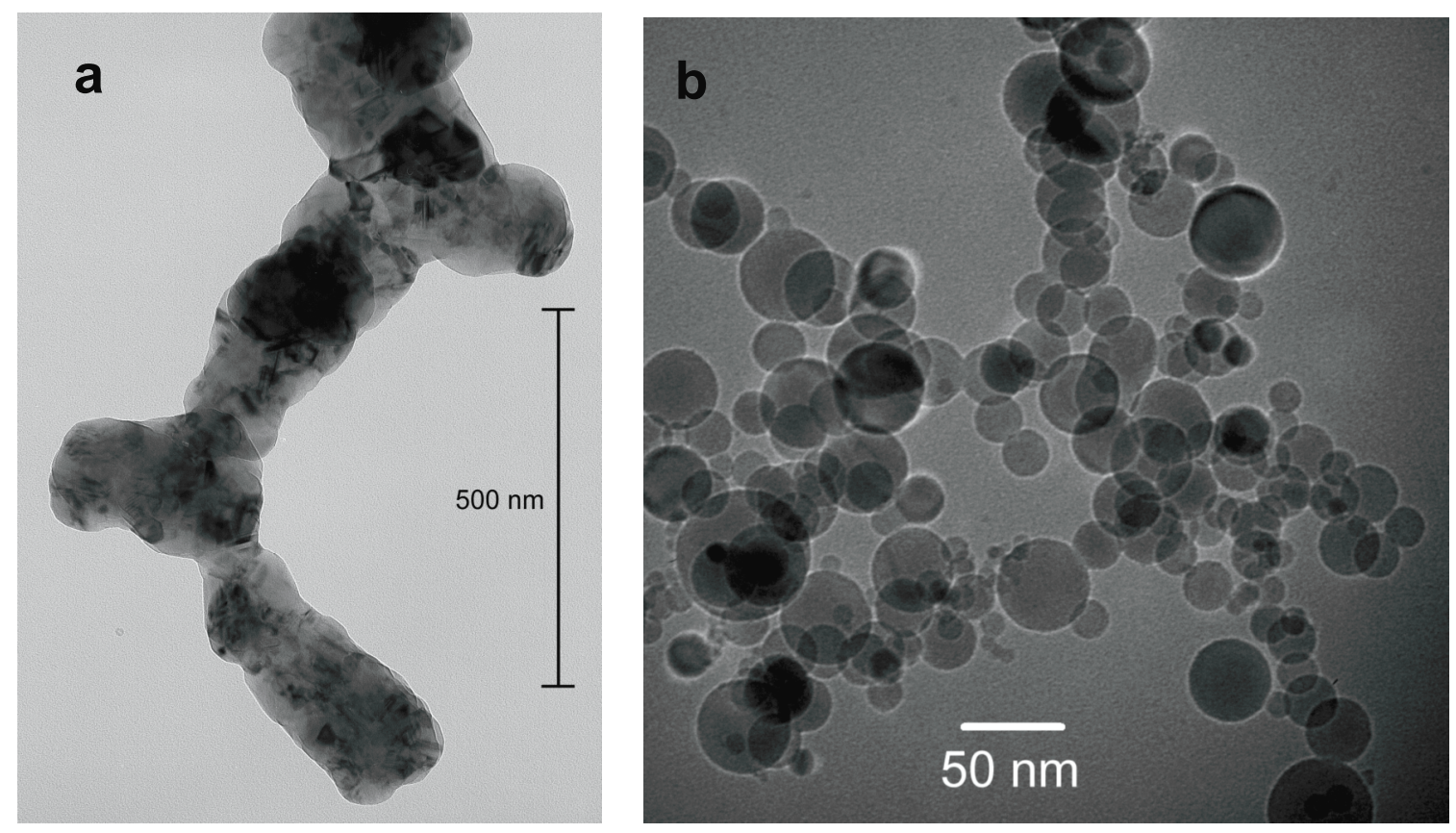

Figure 1: Nanocrystalline silicon from gas phase synthesis produced under thermodynamic control (left) in a hot-wall reactor and under kinetic control (right) in a microwave plasma reactor.

In gas phase synthesis, thermal decomposition and/or chemical reaction of the precursor forms a supersaturated vapor, which is thermodynamically less favored than a condensation and nucleation of single vapor atoms to clusters of several molecules. Depending on the characteristic times for collision and fusion, collision-limited and sintering-limited particles are formed, respectively ${ }^{32}$. Regarding the specific properties required for thermoelectric materials - small particles in the $10 \mathrm{~nm}$ range synthesis conditions have to be adapted in such a way that the characteristic time constants for nucleation, coalescence and sintering are much lower than the characteristic collision time. According to Tsantilis et al. ${ }^{33}$, a third parameter, the cooling rate downstream the reaction chamber, also has an important impact to particle morphology. Agglomeration and formation of aggregates ("hard agglomerates", see figure $2 \mathrm{a}$ ) starts, when the sintering time exceeds the collision time ${ }^{34}$, which always will happen during the cooling process but can be suppressed at very high temperature gradients downstream the reaction zone leading to single, spherical particles with very small point contacts ("soft agglomerates", see figure 2b). As a result, the degree of agglomeration can be tuned by the adjustment of temperature and temperature gradient on the one hand and the adjustment of pressure and precursor concentration/vapor density on the other hand. Low precursor concentration 
and low pressure in combination with high temperature and high temperature gradient result in softagglomerated particles, while high pressure and high concentration along with low temperature and shallow temperature gradient leads to hard agglomerates.

The morphology of raw materials used for the synthesis of bulk thermoelectrics is of high importance because of the fact that formation of dense bulk samples is required for high performance. The densification is done by sintering processes which always implicate restructuring and coarsening leading to bigger crystallites. This is in conflict with the requirement of small nanocrystals for low thermal conductivity. Especially materials with covalent bonds like silicon must be heated to very high temperatures just below their melting temperature for successful densification, which is always related to strong coarsening. Two main possibilities related to particle morphology help to overcome this disadvantage: i) reasonable content of very small nanoparticles sizing in the range of a few nanometer which show a significantly reduced melting temperature ${ }^{35,36}$ and ii) addition of small amounts of homogeneously distributed sintering aids ${ }^{37}$. While the first one can be tuned by the particle formation process itself, the latter one is also directly associated to the particle size as it is strongly surfacerelated.

\section{Plasma synthesis of nanoparticles}

A versatile tool for synthesis of soft-agglomerated nanoparticles of all kinds is the plasma process, as it can provide high reactivity, high temperature, high temperature gradients and also high purity. It enables for production of all oxide and non oxide materials, for which respective gaseous or liquid precursors are available. Most important, it allows for a synthesis of nano materials in a continuous process with kinetic control of the particle formation, which makes thermodynamically instable materials accessible.

Plasma synthesis for thermoelectric materials is a developing technique ${ }^{38-41}$ due to the fact that it enables for the formation of highly specific particles in the $10 \mathrm{~nm}$ range. A lot of investigation towards the formation of silicon and germanium nanoparticles within a plasma is carried out from the Kortshagen group ${ }^{31,42,43}$. They utilize a $200 \mathrm{~W}$ radio frequency $(13.56 \mathrm{MHz})$ generator to ignite a capacitively coupled plasma within a quartz glass tube operated at a pressure of a few mbar resulting in non-thermal plasma. Depending on precursor concentration, mass flow and pressure, crystalline 
particles from a few to a few ten $\mathrm{nm}$ are available. Gorla et al. ${ }^{44}$ investigated an argon/silane inductively coupled plasma also operated at $13.56 \mathrm{MHz}$ at a maximum power of $1200 \mathrm{~W}$. This type of non-thermal plasma can be used to either form silicon or germanium thin films by plasma-assisted chemical vapor deposition or to synthesize spherical silicon and germanium nanoparticles, depending on operation conditions. Nozaki et al. ${ }^{45}$ present a microplasma reactor based on a very-highfrequency (VHF, $144 \mathrm{MHz}$ ) process similar to the setup used by the Kortshagen group. The VHF generator is connected to two electrodes to ignite a non-thermal plasma within a glass tube. The apparatus is operated at atmospheric pressure with a power of 15 to $20 \mathrm{~W}$. Silicon nanoparticles sizing from 3 to $15 \mathrm{~nm}$ in diameter can be produced from a mixture of argon, hydrogen and $\mathrm{SiCl}_{4}$.

Direct current (DC) arc discharges are widely used to ignite thermal plasma such as the one used by Rao et al. to synthesize silicon nanoparticles from silicon tetrachloride ${ }^{46}$. Due to thermal decomposition, the precursors induce a supersaturated vapor with subsequent particle formation. The gas/particle mixture is expanded through a nozzle into a water-cooled reactor vessel, quenching the particle formation process. Calorimetric energy balance measurements determined temperatures in the range of a few thousand $\mathrm{K}$ and due to the immediate cooling process, a quench rate greater than $5 \times 10^{6} \mathrm{~K} \mathrm{~s}^{-1}$ was found. Sophisticated sampling techniques have been developed for near real time inline-measurements of particle size distribution and it is found that nanoparticles sizing between a few and hundred $\mathrm{nm}$ in diameter can be achieved depending on pressure and precursor concentration ${ }^{47}$. DC arc discharge enables for the production of a few grams per hour of spherical, nanosized silicon particles, while the steep temperature gradient prevents them from aggregation. It is a well-known and stable technology that is widely used in industry, especially in gas phase processes. Nevertheless, the DC arc based plasma reactor has a strong disadvantage regarding the purity when used for the synthesis of nanoparticles. Due to erosion from the electrodes, traces of the electrode material are always present in the desired product.

With respect to high and controlled purity of materials used for thermoelectrics, we favor a contamination-free reactor design. It should combine the possibilities for high purity synthesis with a high production rate. To this goal, we have implemented a technology based on a microwave-induced plasma. This technology enables to focus the microwave power into a very small volume within a 
quartz glass tube. The plasma can be stabilized in the center of the tube and reaches gas temperatures considerably higher than $1000 \mathrm{~K}$. This is done by focusing the microwave within a cavity, resulting in a small spot inside a quartz glass tube by constructive interference of the incident waves. The energy transferred from the oscillating electrical field to charged particles is inverse proportional to their mass and to the square of the frequency ${ }^{48}$. Hence, free electrons are accelerated by the electrical field of the microwave, whereas the mass of the heavy ions greatly reduces their acceleration. Thus, electrons with their small mass have considerably more kinetic energy than the heavy particles. This makes microwave plasmas non-thermal plasmas, but especially at higher pressure above a few mbar an elevated gas temperature is found due to collisions of accelerated electrons with neutral gas atoms, molecules and their respective ions, transferring a part of their energy.

Simulation of nanoparticle formation and growth within plasmas is a challenging but increasingly used and highly promising tool for a better understanding of the growth process. The plasma energy coupled into a reactive flow creates very complicated non-equilibrium conditions resulting in various difficulties for modeling attempts. The gas phase is partially ionized, i.e. free electrons and ions are present in the system. The heavy and light species can have different temperatures and their detailed reaction kinetics and diffusional behavior is not known. We have developed a very simple model ignoring all the difficulties described above and considering the absorption of microwave energy as a heat source in the flow resulting in an associated temperature increase ${ }^{49}$. This assumption led to reasonable results for the initial particle formation. A very similar attempt was chosen from Shigeta et al. also neglecting different temperatures and charges ${ }^{50}$. They developed a more suitable model for a numerical analysis of the entire growth of binary nanoparticles in thermal plasma synthesis including homogeneous nucleation and heterogeneous condensation processes. Therefore, it is possible to model nanopowders of any composition and particle size distribution also taking into account the freezing point depression of the nanoparticles. For verification of their model, they made a comparison of the calculation and TEM analysis of nanoparticles from different experiments on silicon-based materials 51, 52 and found a surprisingly good agreement between model and experiment. Despite these encouraging results, up to now there is no suitable model taking into account the plasma-specific properties mentioned above, not to talk of much more complicated topics such as plasma chemistry. 
Therefore, the detailed modeling of particle synthesis in plasmas, especially with respect to nonthermal plasmas, is still a challenge.

\section{Microwave plasma reactor}

The experimental setup used for particle formation in our lab is based on a commercially available Cyrannus plasma source produced by iplas company (Germany), connected to a $6 \mathrm{~kW}$ microwave generator. Typical operation parameters are gas flows from 2 to 6 slm argon, 1 to 4 slm hydrogen and 0.01 to $0.150 \mathrm{slm}$ of silane $\left(\mathrm{SiH}_{4}\right)$ at a pressure between 10 to $200 \mathrm{mbar}$. These conditions lead to an output from 0.7 to $10 \mathrm{~g} \mathrm{~h}^{-1}$ of soft-agglomerated, mostly single-crystalline particles, and their size can be tuned between 4 and $50 \mathrm{~nm}$ by adjusting the experimental conditions.
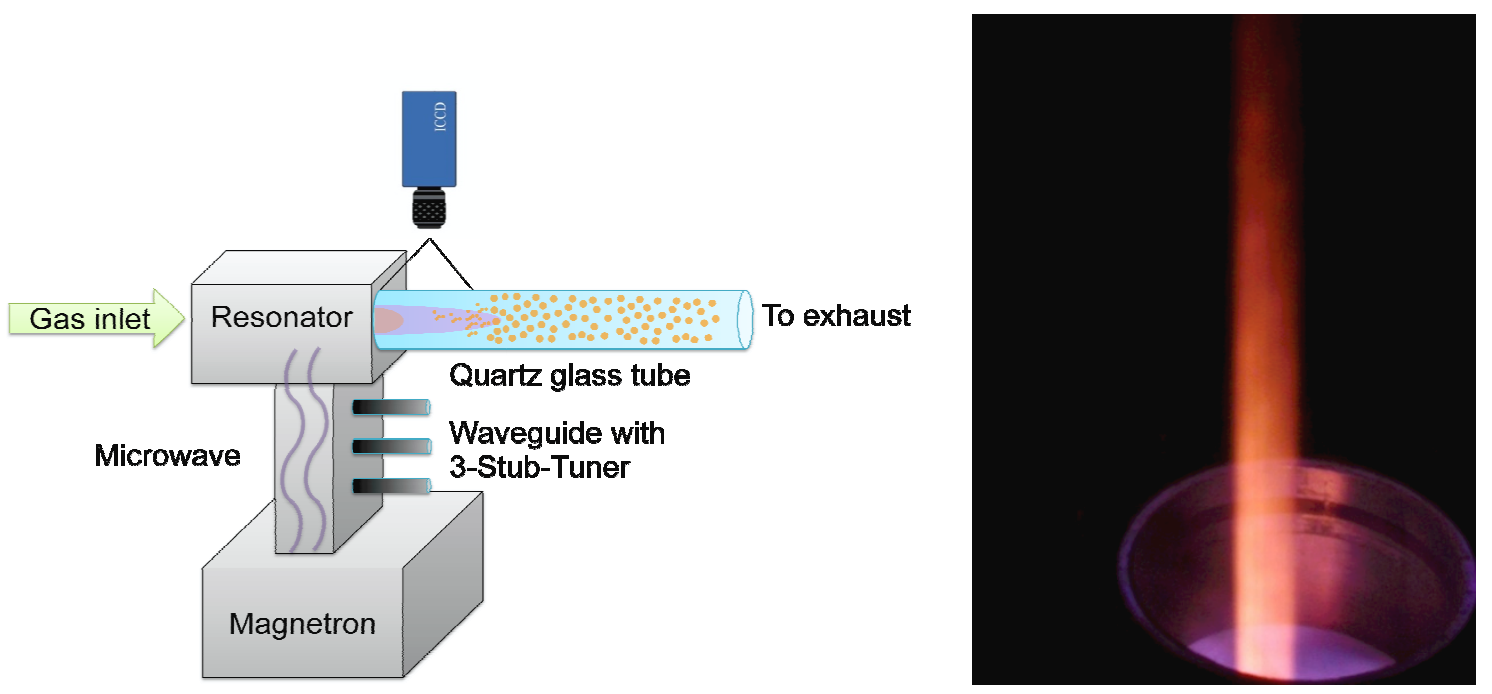

Figure 2: a) Schematic sketch of a lab scale microwave plasma reactor used for nanoparticle synthesis and LIF temperature measurements; b) photograph of the reactor in operation. Note that the plasma itself can hardly be seen (purple luminescence at the bottom), the bright glow originates from the hot nanoparticles leaving the plasma zone and cooling down rapidly.

Optical emission spectroscopy (OES) as well as laser-induced fluorescence (LIF) measurements have been used to measure temperatures for electrons and excited species in microwave plasmas ${ }^{53,54}$. It is found that temperatures can be measured most reliably from laser-induced fluorescence ${ }^{55}$. For the diagnostics of flames, the multiline nitric oxide laser induced fluorescence (NO-LIF) thermometry is a proven technique ${ }^{56}$ and was also applied to plasma reactors used for the formation of silicon 
nanoparticles (see Figure 2). As can be seen from NO-LIF measurements performed to the afterglow of a microwave plasma by means of an excimer laser and an intensified charge coupled device (ICCD) camera, temperatures in the range of 1000 to $2000 \mathrm{~K}$ are found in agreement with results from the literature.

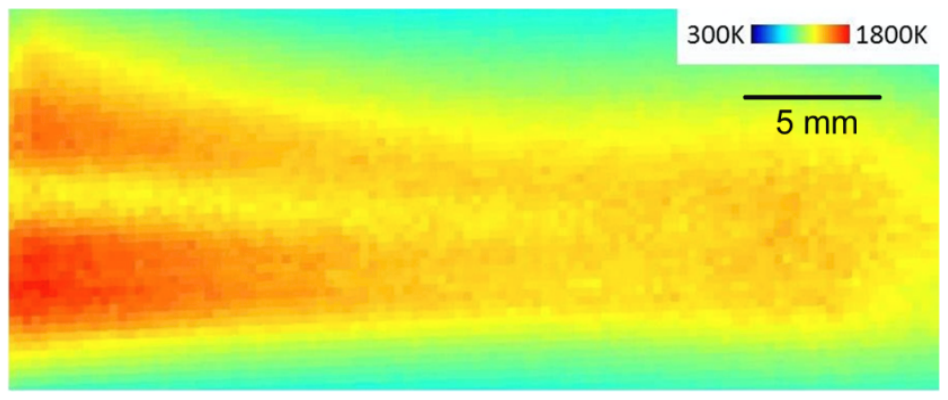

Figure 3: NO-LIF temperature measurements behind the microwave antenna exit at a microwave power of $500 \mathrm{~W}$ and a pressure of $60 \mathrm{mbar}$ (measurements were performed without particle formation)

As in most flow reactor setups, the carrier gases argon and hydrogen as well as the precursor silane are injected through a central nozzle into a quartz glass tube usually leading to some inhomogenities in the gas temperature as can be seen from Figure 3.

\section{In-situ doping of nanoparticles}

For electronic as well as thermoelectric applications, doping of nanoparticles is essential to tailor electrical properties. Although it can be assumed, that solubility of dopants in nanoparticles remain the same as in a bulk material ${ }^{57,58}$, doping of nanoparticles is not trivial. Dopant incorporation, location and efficiency depend on the specific properties of the elements chosen. $\mathrm{N}$-type doping of siliconbased nanoparticles in gas phase synthesis can be realized by adding phosphine $\left(\mathrm{PH}_{3}\right)$ to the gas mixture, while p-type doping can be achieved by adding diborane $\left(\mathrm{B}_{2} \mathrm{H}_{6}\right)$. The maximum solubility of phosphorous in $\mathrm{Si}$ is 2.4 at. $\%$ at $1180^{\circ} \mathrm{C}^{59}$, while the solubility of boron in silicon is found to vary between 0.21 and 3 at. ${ }^{60}$.

Complete decomposition of the respective dopant precursor during plasma synthesis is observed and quantitative incorporation within the nanocrystalline powder is found ${ }^{58,61}$. Nevertheless, only a fraction of the added dopant was homogeneously embedded within the crystalline particles while a reasonable amount segregates at the particle surface. Up to now there is no final understanding 
concerning the incorporation of dopants at high levels much above those usually used for semiconductor application. Self-purification - assisted by short diffusion lengths within the nanoparticles - larger formation energies for substitutional incorporation, strain and mismatching size are some of the points under discussion $57,58,61-64$. However, all investigations dealing with the localization of dopants in nanosized particles from gas phase synthesis find that the mean concentration matches the intended value, while fluctuations are found at the scale of the nanoparticles. Therefore, an almost homogeneous distribution of dopants can be assumed from plasma synthesis of small, nanoscale materials. This finding might be due to the fact, that plasma processes are very fast and usually performed under kinetic control.

\section{Plasma-made nanoparticles}

All plasma gas phase synthesis methods described above are capable to produce mostly spherical and soft-agglomerated nanoparticles. As mentioned above, this is the preferred morphology with respect to further processing by sintering. A typical image of an as-prepared material can be seen from Figure 1b. Despite the utilization of low melting point according to small particle size, we also want to take advantage of the often unwanted formation of a native oxide on the silicon surface. It is a well-known fact, that almost all materials when handled in air show the occurrence of chemically bond hydroxyl groups and oxides on their surface. Due to their high surface-to-volume ratio, this is an important process in surface termination of nanomaterials and can result in a double-digit percentage of concentration with respect to the main composition of the nanomaterial. Silicon when kept in air usually forms a silicon oxide layer with a thickness of 1-2 nm. 


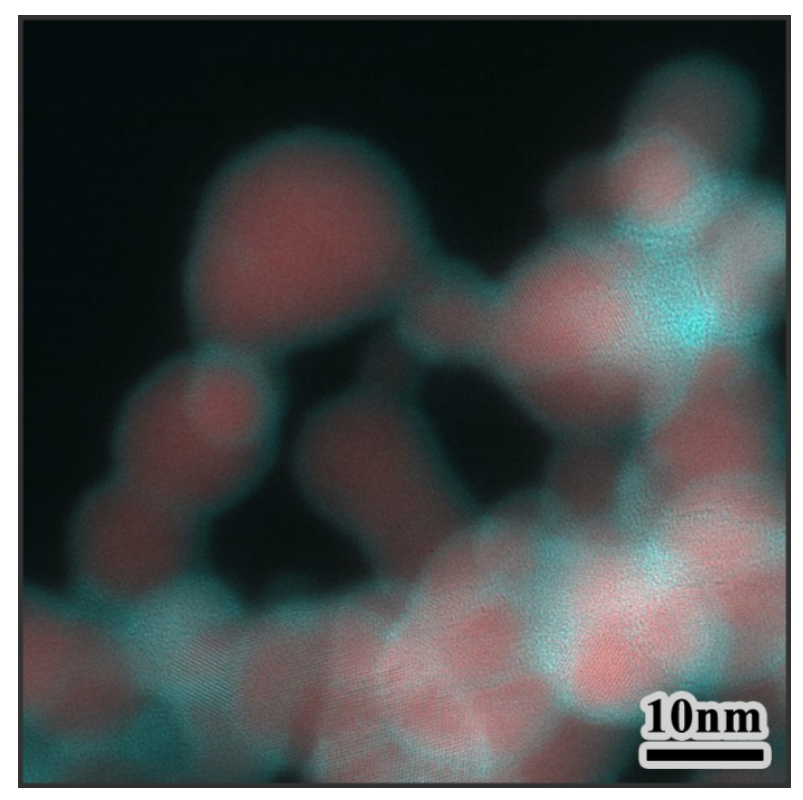

Figure 4: High resolution energy filtered transmission electron microscopy of silicon nanoparticles stored under ambient conditions. Blue colour indicates silicon oxide while silicon is marked in red.

Figure 4 confirms this finding as it clearly indicates that the particle surface is covered with a thin silicon oxide while the core consists of crystalline silicon. It is also found that areas with an initial point contact do not oxidize as much as the free surface and the native oxide forms a common envelope.

\section{Thermoelectrics from plasma-made Si-NPs}

The formation of bulk samples for thermoelectric applications can be done by densification of the doped silicon nanoparticles obtained from the gas phase plasma process. After precompaction to a density of about $50 \%$ and subsequent field assisted sintering ${ }^{65}$, dense pellets can be obtained. As the maximum voltage drop is expected to occur at the grain boundaries between the interconnecting particles, heating and subsequent sintering starts at the particle/particle contacts. Typical heating rates are in the range of $100 \mathrm{~K} \mathrm{~min}^{-1}$ and densification can be achieved within minutes. The density of the resulting pellets was found to vary between $95 \%$ and $98 \%$ of the bulk density of crystalline bulk silicon. 


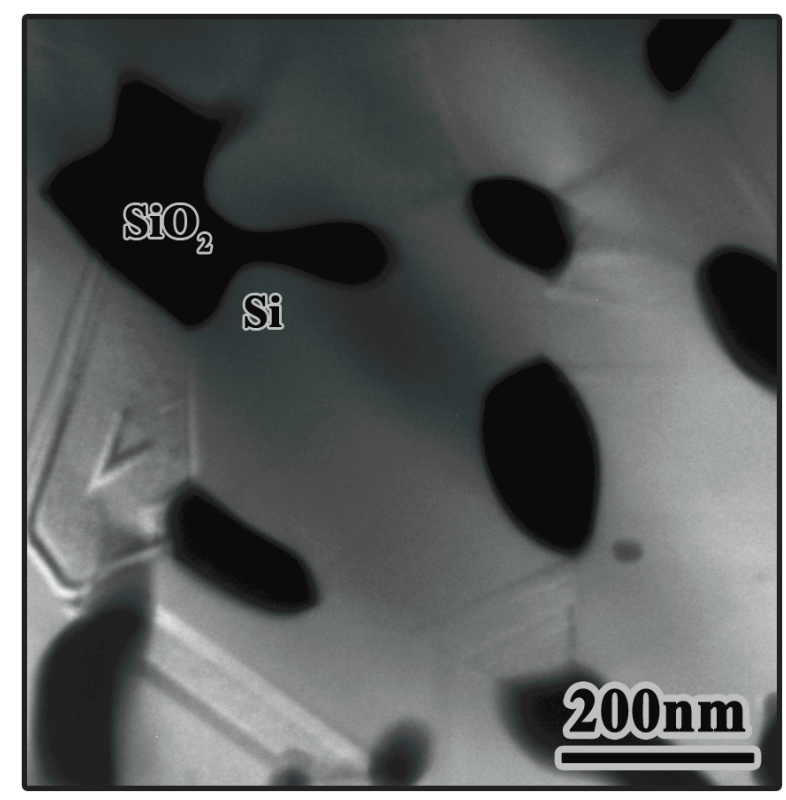

Figure 5: Energy filtered TEM of nanocrystalline silicon, sintered at $1060{ }^{\circ} \mathrm{C}$ making use of the plasmon energy of silicon and silicon dioxide, respectively. Areas consisting of silicon appear bright while areas consisting of silicon dioxide are dark.

The field assisted sintering process takes advantage of two materials properties that are directly related to the particle formation process. i) As is known from the literature the sintering temperature of nanosized silicon is dramatically reduced compared to bulk silicon ${ }^{35,36}$. It was observed that small silicon nanoparticles from the gas-phase sizing $20 \mathrm{~nm}$ and below partially melt and recrystallize immediately starting from about $800{ }^{\circ} \mathrm{C}$ while forming slightly bigger crystallites compared to their initial size. This process is especially observed for the very small crystallites that are "eaten" by the bigger crystallites. ii) As described above, plasma synthesis of the silicon nanoparticles results in spherical particles with weak point contacts due to van der Waals forces leading to a softagglomerated powder. The handling of these materials under ambient conditions results in a thin native oxide shell surrounding the crystalline silicon core. During sintering, this native oxide strongly supports the densification of the pressed pellets at surprisingly low temperatures due to a viscous flow sintering of the oxide ${ }^{66}$. The silicon oxide precipitates while a three-dimensional interconnecting silicon network is formed as can be seen from Figure 5. X-ray diffraction of as-prepared and sintered materials (not shown) reveals that the mean crystallite diameter is only little affected during sintering and remains almost constant. As a result, almost dense pellets are received at a temperature $350{ }^{\circ} \mathrm{C}$ 
below the melting temperature of silicon which is surprising all the more considering the fact, that silicon shows very poor sintering due to the covalent nature of its bonds. Additionally, we are able to synthesize thermoelectrics by keeping the initial crystallite size that can be adjusted by the plasma process parameters.

\section{Thermoelectric properties}

The samples used for the characterization of the thermoelectric properties were made from silicon nanoparticles with a nominal dopant concentration of $5 * 10^{20} \mathrm{~cm}^{-3}$. The Seebeck coefficient was measured by a reference method from MMR Technologies (Micro Miniature Refrigerators) using a constantan wire as reference material while the specific electrical conductivity used to calculate the charge carrier density was determined in van der Pauw geometry ${ }^{67}$. Thermal conductivity was investigated using a laser flash method with LFA 457 MicroFlash from NETZSCH Gerätebau GmbH, while ZT was measured applying a modified Harman method ${ }^{68}$.

The investigations concerning the characterization of charge carrier density reveal that after sintering about one third of the phosphorous atoms is electrically activated within the silicon, while the main amount is found to be accumulated in the silicon oxide forming a phosphorous glass. For all samples investigated, typical values found for the Seebeck coefficient are around 100 to $150 \mu \mathrm{V} \mathrm{K}^{-1}$, and the electrical conductivity was measured between 200 and $900 \mathrm{~S} \mathrm{~cm}^{-1}$, depending on the density of the samples. This indicates that the Seebeck coefficient and electrical conductivity are similar to literature values found for single crystalline silicon and are only weakly affected by the nanostructure of the samples as expected. In contrast, the thermal conductivity of our nanostructured samples is found to be typically between $10 \mathrm{~W} \mathrm{~m}^{-1} \mathrm{~K}^{-1}$ and $20 \mathrm{~W} \mathrm{~m}^{-1} \mathrm{~K}^{-1}$, and therewith about one order of magnitude lower than that of single crystalline bulk silicon and about five times lower compared to polycrystalline silicon, see red dots in Figure 6a. 

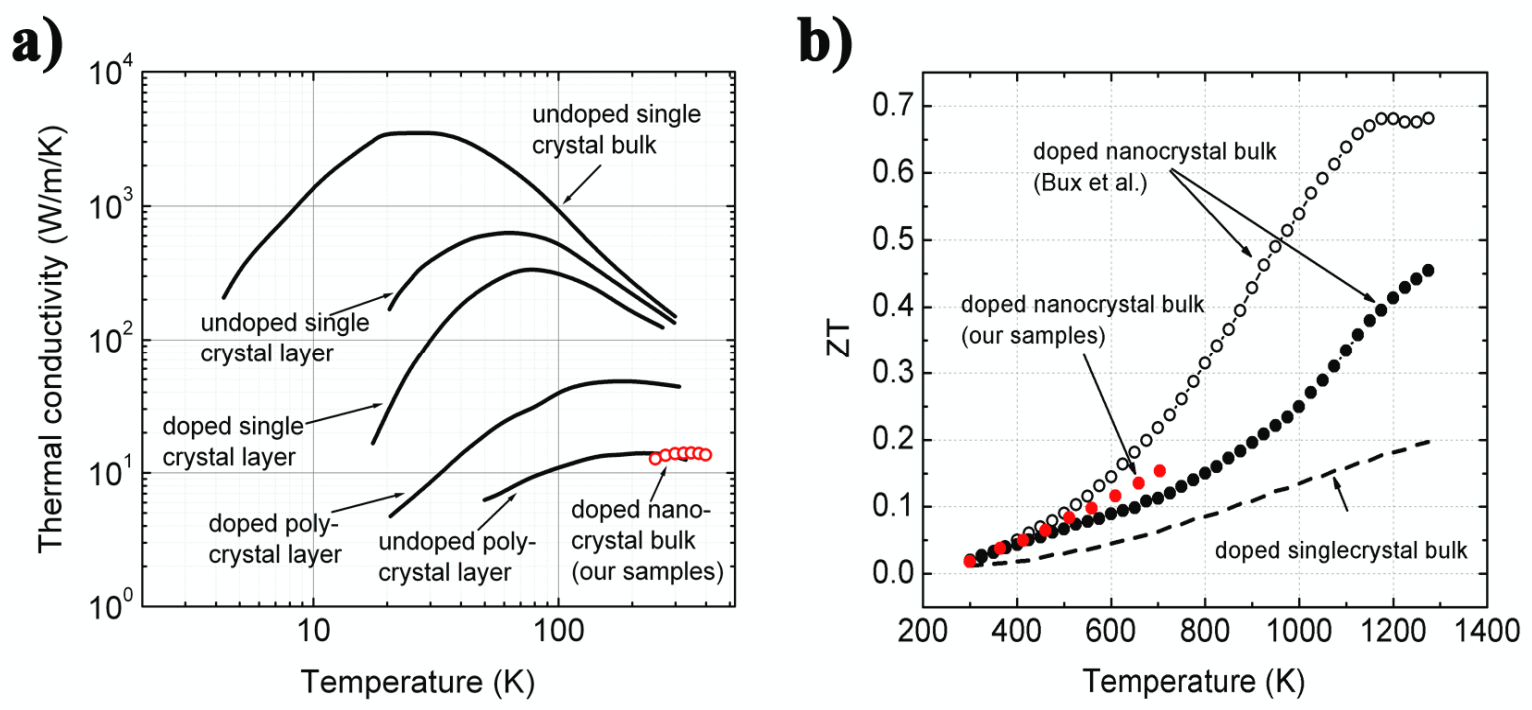

Figure 6: Comparison of the thermoelectric properties of our field assisted sintered nanocrystalline silicon to literature data; a) Temperature-dependent thermal conductivity of different types of silicon, composed after a review by D. Cahill et al. ${ }^{69}$. The value of around $14 \mathrm{~W} \mathrm{~m}^{-1} \mathrm{~K}^{-1}$ at RT for our nanocrystalline bulk silicon is one order of magnitude lower compared to single crystalline bulk silicon; b) Comparison of the figure of merit shown for single crystalline bulk silicon, samples prepared by Bux et al. ${ }^{21}$, The different curves show experiments performed on bulk silicon (dashed line), two samples with different ball milling time and silicon-ball ratio, respectively (open and closed circles), and-our samples prepared from plasma-synthesized silicon (red dots).

Being slightly above $10 \mathrm{~W} \mathrm{~m}^{-1} \mathrm{~K}^{-1}$, the thermal conductivity of our samples is on the same level as undoped polycrystalline layer silicon shown in Figure 6a where a comparison of thermal conductivities of different types of silicon after a review by Cahill et al. is presented ${ }^{69}$. To derive values for the figure of merit ZT, a modified Harman method was applied to our field assisted sintered samples at temperatures between 300 and $700 \mathrm{~K}$. As can be seen from Figure 6b, our samples can compete with samples presented by Bux et al. prepared from ball-milled silicon and phosphorous powder ${ }^{21}$. Besides using a different technique for sample preparation, they also used a more than tenfold higher dopant concentration at around $5^{*} 10^{21} \mathrm{~cm}^{-3}$ within their samples, which may be the main origin of the difference in ZT between their and our samples. 


\section{How to proceed?}

As has been shown, gas phase plasma processing of gaseous precursors towards soft-agglomerated nanopowders is a reasonable and successful way to nanostructured thermoelectrics and enables for a one-step formation of raw materials required for the production of thermoelectric devices. Nevertheless, a combination of the technologies described here with established plasma powder processes such as restructuring approaches as described by Shigeta et al. are highly promising ${ }^{52}$. Regarding the formation of nanocomposites e.g. consisting of SiGe with embedded nanocrystalline silicides as proposed by the group of Shakouri ${ }^{13}$, one might think about the combination of pure gas phase processes (for the formation of highly doped $\mathrm{Si} / \mathrm{SiGe}$ ) with plasma spray technologies based on the reconstruction of (micro) crystalline silicides leading to the respective composite. To our opinion, plasma spray technologies are highly favored for such an approach due to the fact that they have been adapted to a lot of different atmospheres including inert conditions. As nanoparticle formation as well as reconstruction are well-established plasma spray technologies operated at production scale ${ }^{70-72}$, combination with gas phase microwave plasma reactors in the $100 \mathrm{~kW}$ range is highly promising. Nevertheless, a lot of technological challenges have to be overcome such as clogging, contamination and particle deposition due to thermophoresis.

\section{Acknowledgements}

Financial support by the German Research Foundation (DFG) within the Priority Program on nanoscaled thermoelectric materials, SPP 1386, and within the Collaborative Research Center on nanoparticles from the gas phase, SFB 445, is gratefully acknowledged. Financial support by the European Union and the Ministry of Economic Affairs and Energy of the State North RhineWestphalia in Germany in the frame of an Objective 2 Programme (European Regional Development Fund, ERDF) and a Young Investigator Grant is gratefully acknowledged. 
1. G. L. Bennet, presented at the 4th International Energy Conversion Engineering Conference and Exhibit (IECEC), San Diego, CA, 2006 (unpublished).

2. U. S. Congress, edited by O. o. T. Assessment (Washington DC, 1994).

3. J. B. Heywood, Internal Combustion Engine Fundamentals. (McGraw-Hill Inc., 1988).

4. D. Singh, J. Y. Murthy and T. S. Fisher, in ASME/Pacific Rim Technical Conference and Exhibition on Packaging and Integration of Electronic and Photonic Systems, MEMS and NEMS (InterPACK 09), (San Francisco CA, 2009).

5. K. F. Hsu, S. Loo, F. Guo, W. Chen, J. S. Dyck, C. Uher, T. Hogan, E. K. Polychroniadis and M. G. Kanatzidis, Science 303 (5659), 818-821 (2004).

6. $\quad$ N. Peranio and O. Eibl, Journal of Applied Physics 103 (2), 024314-024319 (2008).

7. W. Kim, J. Zide, A. Gossard, D. Klenov, S. Stemmer, A. Shakouri and A. Majumdar, Physical Review Letters 96 (4), 045901-045904 (2006).

8. B. A. Cook, M. J. Kramer, X. Wei, J. L. Harringa and E. M. Levin, Journal of Applied Physics 101, 053715 (2007).

9. W. Schweika, R. P. Hermann, M. Prager, J. Perßon and V. Keppens, Physical Review Letters 99, 125501 (2007).

10. G. S. Nolas, J. L. Cohn and G. A. Slack, Physical Review B 58 (1), 164 (1998).

11. A. M. Guloy, R. Ramlau, Z. Tang, W. Schnelle, M. Baitinger and Y. Grin, Nature 443 (7109), 320-323 (2006).

12. G. A. Slack and M. A. Hussain, Journal of Applied Physics 70 (5), 2694-2718 (1991).

13. N. Mingo, D. Hauser, N. P. Kobayashi, M. Plissonier and A. Shakouri, Nano Letters 9 (2), 711-715 (2009).

14. G. H. Zhu, H. Lee, Y. C. Lan, X. W. Wang, G. Joshi, D. Z. Wang, J. Yang, D. Vashaee, H. Guilbert, A. Pillitteri, M. S. Dresselhaus, G. Chen and Z. F. Ren, Physical Review Letters 102 (19), 196803-196804 (2009).

15. N. Scoville, C. Bajgar, J. Rolfe, J.-P. Fleurial and J. Vandersande, Nanostructured Materials 5 (2), 207-223 (1995).

16. A. I. Boukai, Y. Bunimovich, J. Tahir-Kheli, J.-K. Yu, W. A. Goddard Iii and J. R. Heath, Nature 451 (7175), 168-171 (2008).

17. A. I. Hochbaum, R. Chen, R. D. Delgado, W. Liang, E. C. Garnett, M. Najarian, A. Majumdar and P. Yang, Nature 451 (7175), 163-167 (2008).

18. T. C. Harman, P. J. Taylor, M. P. Walsh and B. E. LaForge, Science 27, 2229-2232 (2002).

19. G. J. Snyder and E. S. Toberer, Nature Materials 7 (2), 105-114 (2008).

20. X. W. Wang, H. Lee, Y. C. Lan, G. H. Zhu, G. Joshi, D. Z. Wang, J. Yang, A. J. Muto, M. Y. Tang, J. Klatsky, S. Song, M. S. Dresselhaus, G. Chen and Z. F. Ren, Applied Physics Letters 93 (19), 193121-193123 (2008).

21. S. K. Bux, R. G. Blair, P. K. Gogna, H. Lee, G. Chen, M. S. Dresselhaus, R. B. Kaner and J.P. Fleurial, Advanced Materials 19 (15), 2445-2452 (2009).

22. H. R. Shanks, P. D. Maycock, P. H. Sidles and G. C. Danielson, Physical Review 130 (5), 1743 (1963).

23. J. P. Dismukes, L. Ekstrom, F. Steigmeier, I. Kudman and D. S. Beers, Journal of Applied Physics 35 (10), 2899-2907 (1964).

24. D. M. Rowe, V. S. Shukla and N. Savvides, Nature 290 (5809), 765-766 (1981).

25. F. Antoni, E. Fogarassy, C. Fuchs, J. J. Grob, B. Prevot and J. P. Stoquert, Applied Physics Letters 67 (14), 2072-2074 (1995).

26. C. B. Murray, C. R. Kagan and M. G. Bawendi, Annual Review Of Materials Science 30, 545$610(2000)$.

27. F. E. Kruis, H. Fissan and A. Peled, Journal Of Aerosol Science 29 (5-6), 511-535 (1998).

28. A. Gurav, T. Kodas, T. Pluym and Y. Xiong, Aerosol Science And Technology 19 (4), $411-$ 452 (1993).

29. M. T. Swihart, Current opinion in Colloid and Interface Science 8, 127-133 (2003).

30. H. Wiggers, R. Starke and P. Roth, Chemical Engineering \& Technology 24, 261 (2001).

31. R. Gresback, Z. Holman and U. Kortshagen, Applied Physics Letters 91 (9) (2007). 
32. M. K. Wu, R. S. Windeler, C. K. R. Steiner, T. Bors and S. K. Friedlander, Aerosol Science And Technology 19 (4), 527-548 (1993).

33. S. Tsantilis and S. E. Pratsinis, Langmuir 20, 5933-5939 (2004).

34. K. E. J. Lehtinen, R. S. Windeler and S. K. Friedlander, Journal of Aerosol Science 27 (6), 883-896 (1996).

35. A. N. Goldstein, Applied Physics A-Materials Science \& Processing 62 (1), 33-37 (1996).

36. G. Schierning, R. Theissmann, H. Wiggers, D. Sudfeld, A. Ebbers, D. Franke, V. T. Witusiewicz and M. Apel, Journal of Applied Physics 103 (8), 084305 (2008).

37. L. W. Wang, W. M. Sigmund, S. Roy and F. Aldinger, Journal Of Materials Research 14 (12), 4562-4569 (1999).

38. O. Fukumasa, Thin Solid Films 390 (1-2), 37-43 (2001).

39. F. Brochin, B. Lenoir, X. Devaux, R. Martin-Lopez and H. Scherrer, Journal Of Applied Physics 88 (6), 3269-3275 (2000).

40. T. Tokiai, T. Uesugi, M. Nosaka, A. Hirayama, K. Ito and K. Koumoto, Journal Of Materials Science 32 (11), 3007-3011 (1997).

41. X. K. Duan, J. Y. Yang, W. Zhong, W. Zhu, S. Q. Bao and X. A. Fan, Powder Technology 172 (2), 63-66 (2007).

42. L. Mangolini, E. Thimsen and U. Kortshagen, Nano Letters 5 (4), 655-659 (2005).

43. A. Bapat, M. Gatti, Y. P. Ding, S. A. Campbell and U. Kortshagen, Journal of Physics D: Applied Physics 40, 2247-2257 (2007).

44. C. R. Gorla, S. Liang, G. S. Tompa, W. E. Mayo and Y. Lu, Journal Of Vacuum Science \& Technology A-Vacuum Surfaces And Films 15 (3), 860-864 (1997).

45. T. Nozaki, K. Sasaki, T. Ogino, D. Asahi and K. Okazaki, Journal of Thermal Science and Technology 2 (2), 192-199 (2007).

46. N. Rao, S. Girshick, J. Herberlein, P. McMurry, S. Jones, D. Hansen and B. Micheel, Plasma Chemistry and Plasma Processing 15 (4), 581-606 (1995).

47. X. Wang, J. Hafiz, R. Mukherjee, T. Renault, J. Heberlein, S. L. Girshick and P. H. McMurry, Plasma Chemistry and Plasma Processing 25 (5), 439-453 (2005).

48. D. Vollath and D. V. Szabó, Journal of Nanoparticle Research 1, 235-242 (1999).

49. B. Giesen, H. Wiggers, A. Kowalik and P. Roth, Journal Of Nanoparticle Research 7 (1), 2941 (2005).

50. M. Shigeta and T. Watanabe, Journal of Applied Physics 108 (4), 043306-043315 (2010).

51. T. Watanabe and H. Okumiya, Science and Technology of Advanced Materials 5 (5-6), 639 (2004).

52. M. Shigeta and T. Watanabe, Journal Of Physics D-Applied Physics 40 (8), 2407-2419 (2007).

53. C. Bohm and J. Perrin, Journal Of Physics D-Applied Physics 24 (6), 865-881 (1991).

54. Y. Kabouzi, M. D. Calzada, M. Moisan, K. C. Tran and C. Trassy, Journal Of Applied Physics 91 (3), 1008-1019 (2002).

55. G. P. Davis and R. A. Gottscho, Journal Of Applied Physics 54 (6), 3080-3086 (1983).

56. W. G. Bessler and C. Schulz, Appl. Phys. B 78, 519-533 (2004).

57. M. Perego, C. Bonafos and M. Fanciulli, Nanotechnology 21 (2), 025602 (2010).

58. X. D. Pi, R. Gresback, R. W. Liptak, S. A. Campbell and U. Kortshagen, Applied Physics Letters 92, 123102 (2008).

59. R. Olesinski, N. Kanani and G. Abbaschian, Journal of Phase Equilibria 6 (2), 130-133 (1985).

60. R. Olesinski and G. Abbaschian, Journal of Phase Equilibria 5 (5), 478-484 (1984).

61. A. R. Stegner, R. N. Pereira, R. Lechner, K. Klein, H. Wiggers, M. Stutzmann and M. S. Brandt, Physical Review B 80 (16), 165326 (2009).

62. G. Cantele, E. Degoli, E. Luppi, R. Magri, D. Ninno, G. Iadonisi and S. Ossicini, Physical Review B 72 (11), 113303 (2005).

63. S. C. Erwin, L. Zu, M. I. Haftel, A. L. Efros, T. A. Kennedy and D. J. Norris, Nature 436 (7047), 91-94 (2005).

64. D. J. Norris, A. L. Efros and S. C. Erwin, Science 319 (5871), 1776-1779 (2008).

65. J. R. Groza and A. Zavaliangos, Materials Science and Engineering A-Structural Materials Properties Microstructure and Processing 287 (2), 171-177 (2000). 
66. C. J. Brinker and G. W. Scherer, Sol-gel science: the physics and chemistry of sol-gel processing. (Academic Press Inc., San Diego, 1990).

67. L. J. v. d. Pauw, Philips Research Reports 13, 1-9 (1958).

68. T. C. Harman, Journal of Applied Physics 29 (9), 1373-1374 (1958).

69. D. G. Cahill, W. K. Ford, K. E. Goodson, G. D. Mahan, A. Majumdar, H. J. Maris, R. Merlin and S. R. Phillpot, Journal of Applied Physics 93 (2), 793-818 (2003).

70. B. H. Kear, J. Colaizzi, W. E. Mayo and S. C. Liao, Scripta Materialia 44 (8-9), 2065-2068 (2001).

71. J. Karthikeyan, C. C. Berndt, J. Tikkanen, S. Reddy and H. Herman, Materials Science And Engineering A-Structural Materials Properties Microstructure And Processing 238 (2), 275286 (1997).

72. M. I. Boulos, High Temperature Material Processes 1 (1), 17-39 (1997). 